\title{
Visitando la CiUdad de Tianshui Una mirada a la cultura marcial de la Ruta de la SEDA DEL NorTe DE ChINA
}

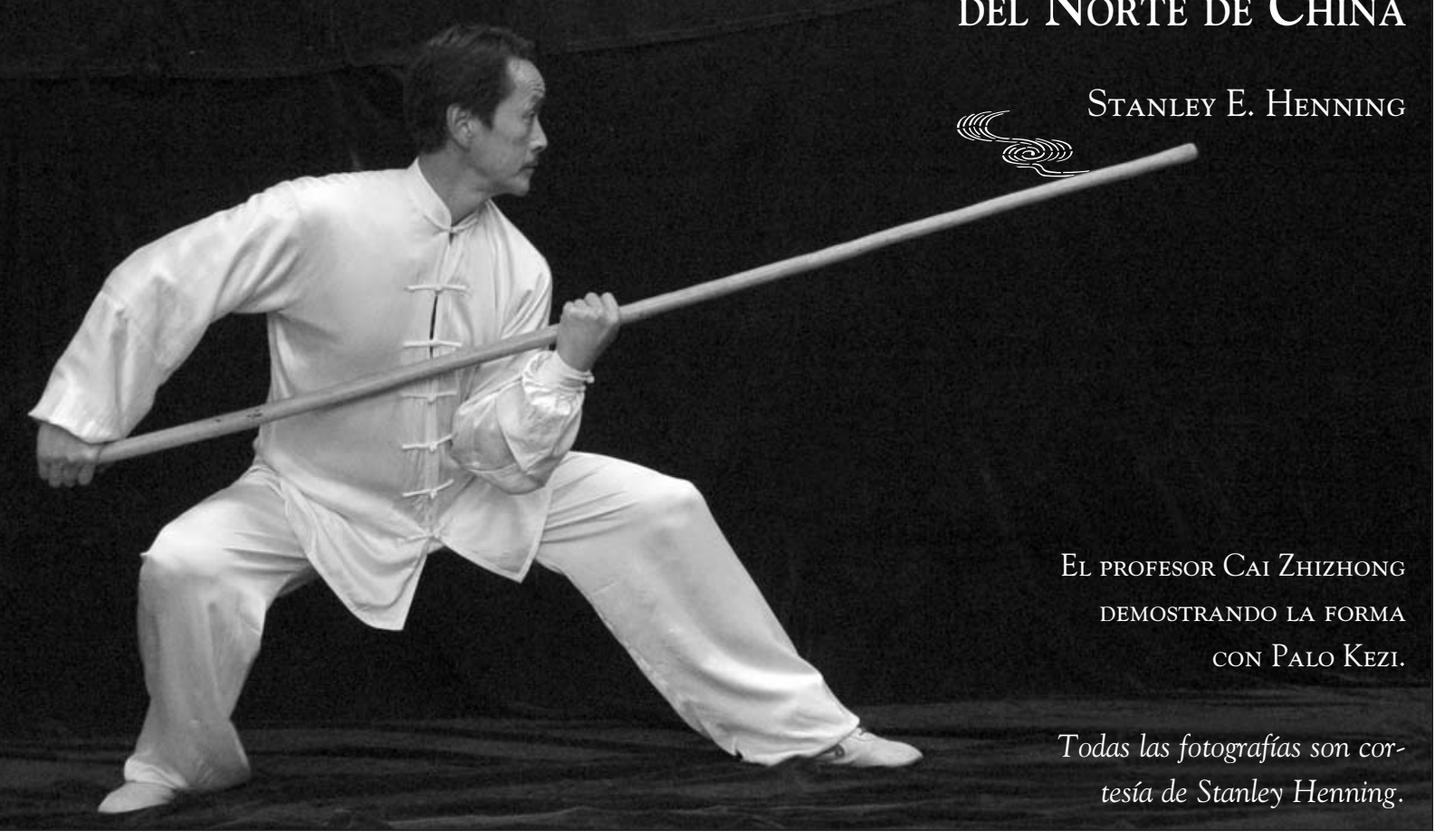

Fu Xi SOsteniendo EL EMBLEMA TAIJI-BAgUA, en el Templo Fu Xi de

TIANSHUI.

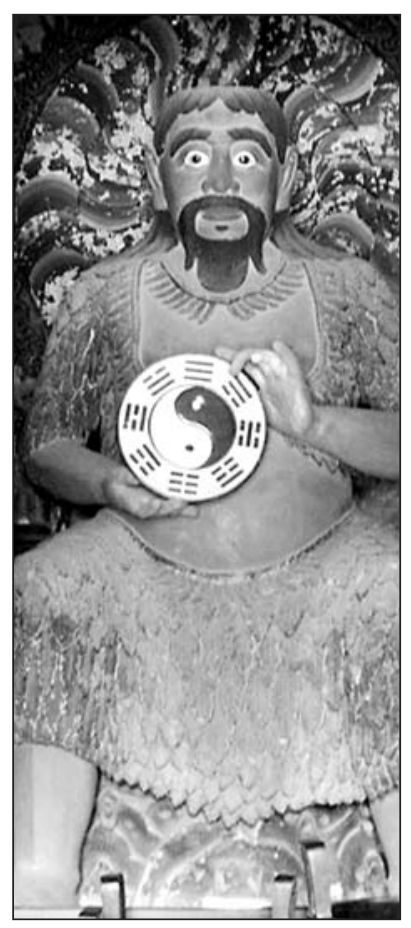

64

\section{Resumen}

La ciudad de Tianshui, situada en el antiguo centro cultural de China en la provincia de Gansu, comprende una amplia zona rural conocida como Qinzhou. Esta zona alberga núcleos que conservan la cultura de las artes marciales tradicionales, permitiendo a uno saborear el pasado en el presente, incluso a pesar del desarrollo económico chino sin precedentes y los cambios sociales que se han producido durante los últimos años. La imagen que se describe en este breve artículo se basa en las visitas del autor a Tianshui, la más reciente en el 2007, en conversaciones in situ con el profesor Cai Zhizhong, que enseña artes marciales en el programa de educación física del Colegio Normal de Tianshui, y en los escritos del profesor Cai sobre la materia. Puesto que la modernización que está teniendo lugar en China no puede ayudar, pero sí tiene influencia en las prácticas de las artes marciales tradicionales de Tianshui, uno vuelve esperanzado de que la fuerte conciencia histórica y el sentimiento de orgullo cultural exhibido por los residentes de esta zona asegurarán un papel continuista para las artes marciales tradicionales de Tianshui.

is visitas a Tianshui en 2002 y 2007 me hicieron darme cuenta no sólo del desarrollo V sin precedentes que está teniendo lugar en China desde que Deng Xiaoping impulsase la Política de Puertas Abiertas en 1978, sino también del resurgimiento del orgullo cultural, incluyendo la preocupación por las artes marciales que son la forma de cultura física tradicional china más extendida. Quizás ningún otro lugar representa este orgullo y preocupación como Tianshui, situada a lo largo de la Ruta de la Seda, en lo profundo del corazón geográfico de la antigua civilización Han, así como en la intersección de varias minorías étnicas.

Los lugares históricos esparcidos por la zona son un recuerdo constante para los residentes de sus lazos con un pasado glorioso. Entre estos, el Templo Fuxi de Tianshui, 
una estructura del período Ming construida por primera vez en 1490, es probablemente el símbolo más significativo de la cultura china centralizada de los Han, por su situación y por albergar la imagen de Fu Xi -el padre legendario de la estirpe china de los Han, sosteniendo entre sus manos el símbolo Taiji rodeado de ocho trigramas (bagua), reflejando una visión universal con fuerte influencia taoísta. No se ahorraron gastos cuando el antiguo presidente chino Jiang Zemin vino aquí para presentar sus respetos en 1992. Se construyó una amplia plaza de piedra, con el símbolo del Taiji-Bagua tallado en su superficie, y una columna con el diseño de un gran dragón y un fénix a la izquierda, para dar a la entrada al templo, reflejando así una continuidad inconfundible de las tendencias socio-culturales tradicionales.

Además del templo $\mathrm{Fu} \mathrm{Xi}$, en la zona hay varios monasterios budistas, esculturas en cuevas/acantilados (el más famoso de los cuales es el monte Maiji), y templos taoístas. Tianshui también fue el hogar de Li Guang ( ? -119 a.C.), uno de los generales chinos más famosos, llamado el "General Volador". Fue conocido por su destreza en el tiro con arco (la leyenda dice que lanzó una flecha a través de una piedra que se parecía a un tigre agazapado) y por su liderazgo en la lucha contra los nómadas Xiongnu. Desde los primeros registros, el tiro con arco fue el arte marcial chino más estimado y una de las Seis Artes (escritura, cálculo, música, ritos, tiro con arco y manejo de carros) que componían la educación confuciana de los líderes. Jiang Wei (202-264 d.C.), un general que sirvió a Zhuge Liang (181-234 d.C.), un estratega político-militar del período de los Tres Reinos del Reino Shu Han (establecido en la vecina Sichuan), también era de la zona de Tianshui. Tianshui fue considerada un paso vulnerable entre los reinos de Shu Han y Wei, por lo que Zhuge Liang había planeado operaciones estratégicas en esta zona e incluso supervisó la construcción de atrincheramientos en Tianshui para detener a las fuerzas contrarias durante sus campañas en el norte contra el Reino de Wei. Estos atrincheramientos son ahora el contenido de un parque temático de Tianshui. Y, como podría esperarse, todas las principales figuras de la novela del s. XV Romance de los Tres Reinos, con la que casi todos los chinos están familiarizados, son descritas como hábiles en varias artes marciales con armas.

Es en este contexto históricamente estimulante donde el profesor Cai Zhizhong, del Colegio Normal de Tianshui, ha publicado un trabajo pionero sobre las diversas artes marciales de la zona de Tianshui titulado Artes Marciales de Tianshui (2003). Situándolas en una perspectiva geográfica, cita un refrán popular generalizando el énfasis de las artes marciales chinas como "puños del sur y piernas del norte, lanza del este y palo del oeste", y apunta que el "palo del oeste" encaja en Tianshui como añillo al dedo, y que, más allá de los varios estilos de boxeo practicados en Tianshui, las formas de palo y de palo corto son más destacables. El profesor Cai también descubre varias manifestaciones de artes marciales tradicionales que ofrecen una perspectiva diferente de la cultura de las artes marciales chinas de la que se encuentra en las historias sobre el Monasterio de Shaolín o en los estilos más conocidos como el Taijiquan y otros influenciados por intelectuales ilustrados. A este respecto, el ejemplo de Tianshui es más representativo de la experiencia rural en las artes marciales en la amplia campiña china. Aunque es bastante cierto que la práctica de las artes marciales existió en la zona durante siglos, las informaciones fiables sobre la mayor parte de estas actividades se remontan a poco antes de finales del s. XIX, y mucho de lo que hoy sabemos se remonta a no mucho antes de que el "Movimiento de Desenterrar y Organizar" las artes marciales comenzase en 1979 y se extendiese durante la década de los 80. Dándose cuenta de que la temprana Revolución Cultural del Gran Proletariado (1966-1976) había apuntado a las artes marciales como albergadoras de características feudales, hecho que resultó en la destrucción de muchos valiosos artefactos y material escrito relacionado con las mismas, las autoridades gubernamentales encargadas de la cultura física iniciaron el "Movimiento de Desenterrar y Organizar" en un intento por recopilar y preservar lo que todavía quedaba. Entre los hallazgos más excitantes de este movimiento estaba la práctica continuada en un pueblo de la zona de Tianshui, el pueblo de la familia Gao, de una forma hasta ahora prácticamente desconocida de lucha con palo llamada Palo Kezi.
VISTA DE LAS TALLAS DEL ACANTILADO BUDISTA EN LA montaña Maiji, Tianshui.

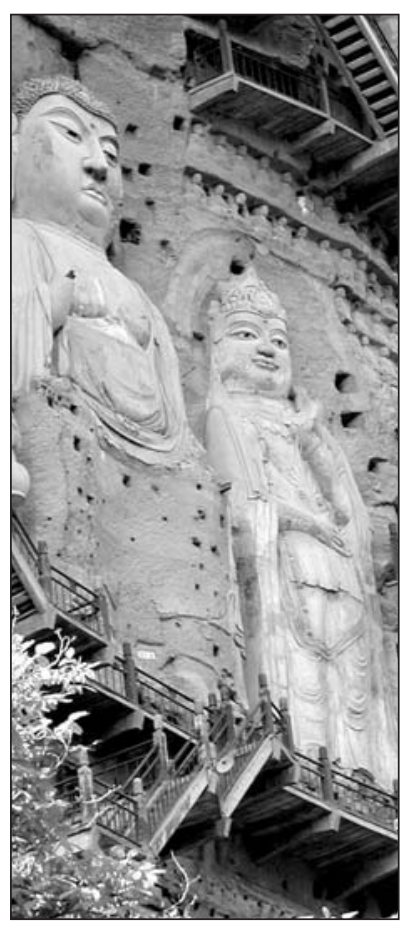




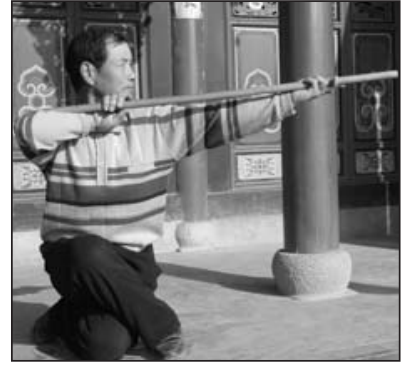

Artista marcial De TIANSHUI DEMOSTRANDO LA FORMA DE PALO DEL LÁTIGO DEL TIGRE BLANCO.

\section{El profesor Cai Zhizhong} FRENTE A LA TUMBA DEL GENERAL Li GuANG.

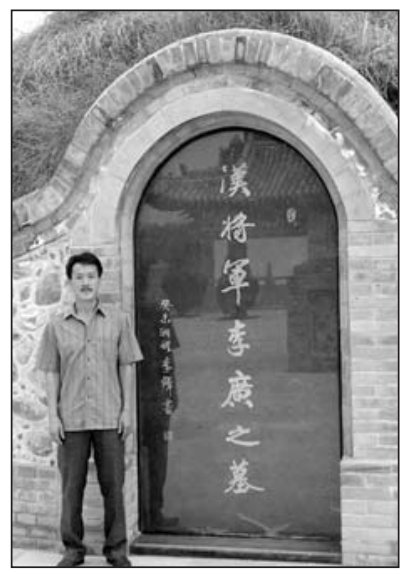

Residente de Tianshui DEMOSTRANDO LA FORMA de PAlo Del Mono. Aquí SOSTIENE UN PALO ENTRE LA CINTURA Y EL PIE DERECHO.

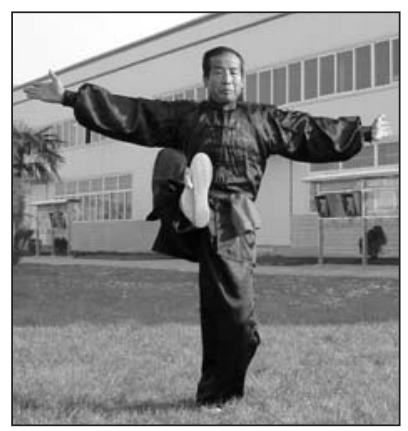

La campiña china está adornada con pequeños pueblos, algunos en terrenos difíciles lo que hizo que fuesen descubiertos en la última parte del s. XX. En lugares como éstos, las artes marciales eran practicadas para la defensa individual y comunitaria (el primer aspecto, práctico), así como para la recreación y el entretenimiento (el aspecto de representación), mientras que el acondicionamiento físico era un aspecto inherente a ellas. Las artes marciales eran introducidas a veces por artistas marciales itinerantes y, en algunos casos como en Tianshui, por individuos asignados a unidades militares locales. La mayor de las cuatro escuelas o grupos principales de artes marciales de Tianshui, llamada la Escuela de las Cuatro Puertas, se desarrolló gracias al entrenamiento que recibieron varios habitantes locales por parte de un oficial en el Campamento Militar Local de Gansu a finales del período Qing (1644-1911). Esta escuela evolucionó en seis sub-escuelas compuestas por diferentes combinaciones de lucha sin armas y rutinas con armas, con características diferenciadas en la ejecución en función de los individuos que se implicaron en ellas. Una de estas sub-escuelas se llamó la Escuela del Campamento Militar por su representante principal, Sun Yanbiao (1880-1975), que sirvió en el ejército. Además de la Escuela de las Cuatro Puertas, existen otras tres escuelas principales en la amplia zona de Tianshui. Una de éstas, la Escuela de la Familia Gao, destaca por su estilo único de combate con palo, "descubierto" durante el "Movimiento de Desenterrar y Organizar".

Los estilos de lucha sin armas practicados por los miembros de varias de las escuelas de artes marciales de Tianshui son numerosos y variados, mostrando una mezcla de rutinas con nombres tales como Boxeo Borracho, Boxeo del Mono, Puño Cañón, Boxeo Yan Qing, Boxeo Hua, Boxeo Jin'gang, Boxeo Hong, Boxeo del Desgarramiento, Nueve Giros y Dieciocho Caídas, y más. Desde 1949, han añadido las artes marciales Tongbei (Completamente Instruido y Marcialmente Preparado) de Ma, introducidas por el profesor y padre de Ma Mingda, Ma Fengtu (1888-1973) y sus hijos, uno de los cuales, Ma Lingda, reside actualmente en Lanzhou, Gansu. Las artes marciales de la Escuela de Ma combinan los estilos tradicionales antiguos de su país de origen, Cangzhou, Hebei, y que vienen a incluir el Tongbi, el Pigua, y el Boxeo Fanzi, las técnicas de patadas del Chuojiao, la Lanza Liuhe, y la espada de dos manos.

Debido a sus aspectos únicos, el Palo Kezi es objeto de estudio en una monografía individual del profesor Cai Zhizhong titulada Investigación del Palo Kezi (2002), ya que es considerado un ejemplo particularmente sobresaliente de las formas de lucha con palo de las artes marciales de Tianshui y, de hecho, es la más sobresaliente de ellas. El célebre historiador de las artes marciales, el profesor Ma Mingda, ofrece una inestimable introducción a la obra, colocando este tesoro popular en perspectiva, tanto históricamente como en términos de estilos tradicionales y de sus técnicas comparativas. De acuerdo con el profesor Ma, las técnicas del Palo Kezi reflejan que es supuestamente un palo del sur, "firme" o de "agarre fuerte", un estilo frecuente de Fujian, Guangdong, Zhejiang, y Hunan, y reflejado en el Clásico de la Espada (Jian Jing) del General del período Ming Yu Dayou (1504-1580). El profesor Ma también señala que la lucha con palo ha sido ampliamente practicada en el noroeste de China (especialmente en Gansu, Qinghai, y Ningxia), donde los locales lo llaman humorísticamente con el término coloquial "leña", y a su práctica como "blandiendo la leña". Sin embargo, el estilo practicado más frecuentemente en esta región ha sido el palo del norte o de "agarre flojo", relacionado con la técnica de la lanza, por lo que todo lo que se sabe es que el Palo Kezi parece haberse introducido en la zona de Tianshui en algún momento de un tiempo lejano. Los aldeanos que practican el Palo Kezi afirman que se decía que fue introducido por un monje shaolin viajero durante la era Qianlong (1736-1795); sin embargo, esta afirmación está sin confirmar. El profesor Ma teoriza que el nombre original para esta forma de palo podría venir de un palo para "golpear/chocar contra" (ke) más que para "modelar/amoldar" (mozi/kezi), como se escribe hoy en día. El término kezi o "caparazón" se pronuncia de forma parecida a "golpear/chocar contra", que se utiliza durante todo el manual del palo del General Yu y que es una característica identificativa de las formas de palo del sur. Por otra parte, el término "caparazón" (supuestamente coloquial para modelar/ 
amoldar), refleja la manera en la que el Palo Kezi se enseña y practica, en grupos de patrones de técnicas más que en largas formas.

El profesor Cai señala que el Palo Kezi se compone de dos métodos principales: punta simple para largas distancias y para los lugares más abiertos, y punta doble para espacios más pequeños y distancias muy próximas. El adiestramiento consta de 66 patrones de movimiento. Describe en detalle estos 66 patrones y demuestra los movimientos clave en fotografías. También describe las 7 fases del entrenamiento práctico: (1) aprender la posición de comienzo de cada patrón y favorecer la memorización con una fórmula oral para aprender todos los patrones; (2) concentrarse en el movimiento final del palo de cada patrón; (3) practicar sobre las zonas de golpeo designadas como objetivo. Practicando el ataque uno tiene que ser preciso, fiero, rápido, y efectivo, por lo que uno tiene que visualizar el cuerpo del oponente como dividido en 10 partes; cada una de las cuales será el objetivo de una o más técnicas; (4) practicar golpeando tanto con la punta simple como con la punta doble; (5) practicar a fondo los usos de cada patrón; (6) practicar tanto los aspectos de ataque como de defensa de cada patrón; y (7) practicar el combate libre o sparring con rapidez, inteligencia, y osadía.

Uno puede ver desde el principio que el Palo Kezi es un sistema individual de combate aparentemente simple, pero realmente es un sistema muy complejo que se ha desarrollado a lo largo del tiempo sobre la base de la experiencia práctica.

Una mirada sobre la amplia variedad de rutinas con y sin armas practicadas por los diversos grupos de artes marciales en la zona de Tianshui, y dándose cuenta de que el contenido de estas rutinas ha cambiado a lo largo del tiempo, revela el hecho de que se han producido intercambios continuos entre los artistas marciales en un constante esfuerzo por mejorar sus habilidades. También es importante darse cuenta de que el papel del palo en este empeño ha sido crucial. Como señala el profesor Ma, durante el periodo Yuan (1271-1368) o Ming (1368-1644), existen evidencias no sólo de ejercicios orientados a la representación, llamados shibang, sino también competiciones de sparring orientadas a la efectividad conocidas como dabang, en las que el Emperador Ming Taizu (13681398) se divertía personalmente actuando como árbitro. Gracias a su flexibilidad de usos en diversas situaciones, el palo fue considerado durante mucho tiempo tanto por los artistas marciales militares como por los civiles como la mejor arma

\begin{tabular}{|c|c|}
\hline \multicolumn{2}{|c|}{ GLOSARIO } \\
\hline bang & 棒 \\
\hline biangan & 鞀杆 \\
\hline Cai Zhizhong & 蔡智忠 \\
\hline dabang & 打棒 \\
\hline Fuxi Miao & 伏羲庙 \\
\hline Gao jia pai & 高家派 \\
\hline Gao jia Wacun & 高家出村 \\
\hline gun & 棍 \\
\hline Jiang Wei & 姜维 \\
\hline kezi gun & 壳子棍 \\
\hline ke & 磕 \\
\hline kezi & 壳子 \\
\hline huoba & 活把 \\
\hline Jian Jing & 《创钡》 \\
\hline koujue & 口就 \\
\hline Li Guang & 李广 \\
\hline Ma Mingda & 馬明连 \\
\hline mozi & 模子 \\
\hline shibang & 使棒 \\
\hline shua chai huo & 要柴火 \\
\hline siba & 死把 \\
\hline simen quan & 四门拳 \\
\hline Sun Yanbiao & 孙彦虎 \\
\hline wajue zhengli yundong & 挖掘整理运动 \\
\hline yingba & 硬把 \\
\hline yu Dayou & 俞大猶 \\
\hline Zhuge Liang & 诸蓠亮 \\
\hline
\end{tabular}
para crear una base firme y completa de habilidades marciales.

Una visita a Tianshui le lleva a uno a través del tiempo a saborear una cultura antigua, mientras que el profesor Cai Zhizhong, a través de sus escritos sobre las artes marciales de Tianshui, ha contribuido de forma significativa a nuestro entendimiento sobre la naturaleza de las artes marciales en la cultura popular de Tianshui y ha abierto la puerta un poco más a nuestro conocimiento sobre el papel de las artes marciales en la China rural y en la cultura china en general.

\section{BIBLIOGRAFÍA}

Cai Zhizhong $\leq(2003)$. Las artes marciales de Tianshui 《天水武术》. Gansu People's Publishers 甘肃人民出版社. Contiene el texto, pero no las fotografías, de Investigación del Palo Kezi.

Cai Zhizhong (2002). Investigación del Palo Kezi《壳子棍研究》. Gansu Educational Publishers 甘肃教育出版社.

Liu YanXiang 刘雁翔 (Agosto 2004). Collected research on Tianshui Three Kingdoms sites, Journal of Tianshui Normal University, 24:4, 74-79.

Qinan County Kezi Gun 壳子棍研究. A documentary about Gao Family Kezi Staff downloaded from: http://www.youtube.com/watch?v=s8Zt553T8fE 